\title{
A folded-sandwich polarization-entangled two-color photon pair source with large tuning capability for applications in hybrid quantum systems
}

\author{
Otto Dietz $^{1}$ - Chris Müller ${ }^{1}$ Thomas Kreiß1 ${ }^{1}$ - Ulrike Herzog ${ }^{1} \cdot$ Tim Kroh $^{1}$ • \\ Andreas Ahlrichs ${ }^{1} \cdot$ Oliver Benson $^{1}$
}

Received: 13 July 2015 / Accepted: 22 September 2015 / Published online: 30 January 2016

(C) Springer-Verlag Berlin Heidelberg 2016

\begin{abstract}
We demonstrate a two-color entangled photon pair source which can be adapted easily to a wide range of wavelength combinations. A Fresnel rhomb as a geometrical quarter-wave plate and a versatile combination of compensation crystals are key components of the source. Entanglement of two photons at the Cs D1 line $(894.3 \mathrm{~nm})$ and at the telecom O-band $(1313.1 \mathrm{~nm})$ with a fidelity of $F=0.753 \pm 0.021$ is demonstrated, and improvements of the setup are discussed.
\end{abstract}

\section{Introduction}

In recent years, there has been an increasing effort to realize and study quantum hybrid systems. These consist of two dissimilar systems - often at different wavelengths-which are in a joint quantum state. Aside from the fundamental insight gained from studying such a peculiar, perhaps multiparticle entangled state, there are also immediate applications in quantum information processing. Entanglement of a stationary and flying qubit, i.e., an electronic state with a long coherence time and a photon, respectively, represents a coherent quantum interface. Such interfaces are mandatory components of a quantum repeater [1], where entanglement has to be established between distant nodes. Experimental realizations demonstrated entanglement between a photon

This paper is part of the topical collection "Quantum Repeaters: From Components to Strategies" guest edited by Manfred Bayer, Christoph Becher and Peter van Loock.

Otto Dietz

otto.dietz@physik.hu-berlin.de

1 Nano-Optics Group, Institut für Physik, HumboldtUniversität zu Berlin, Berlin, Germany and a stationary state in atoms [2], ions [3], semiconductor quantum dots [4], color defect centers [5] and even superconducting qubits [6]. A Bell measurement on two photons of each of two such states would then immediately establish entanglement between two stationary, possibly dissimilar states [7, 8]. However, two dissimilar systems may emit light at vastly different wavelengths. It is not possible to entangle such systems with different transition energies via a Bell state measurement. One possible solution of this problem is to use a two-color pair of entangled photons, which are color-matched to these two different transitions as shown in Fig. 1a. The two-color photon pair source mediates entanglement between two dissimilar wavelengths. In the same manner, it can convert the wavelength of flying qubits as shown in Fig. 1b. This is especially helpful if entanglement should be established over long distances, e.g., via optical fibers in the telecom band. The key building block in these two applications is a source of non-degenerate entangled photons. Especially when working with systems whose wavelengths are hundreds of nanometers apart, such a source has to generate highly non-degenerate photons. In contrast to previous highly non-degenerate sources [9], our source introduces wavelength-insensitive components and is thus in principle widely tunable. This is another important requirement when working with a variety of different systems in hybrid systems, in particular with systems that may vary in time or from experiment to experiment.

Up to now, the brightest and most practical sources of entangled photon pairs rely on spontaneous parametric down-conversion in nonlinear crystals [10]. With appropriate phase matching, two-color sources with photons at different wavelengths can be realized $[9,11,12]$. For subsequent fiber coupling, collinear down-conversion schemes are most convenient. Furthermore, the collinear alignment enables further integration and can be used in a monolithic design [12]. 

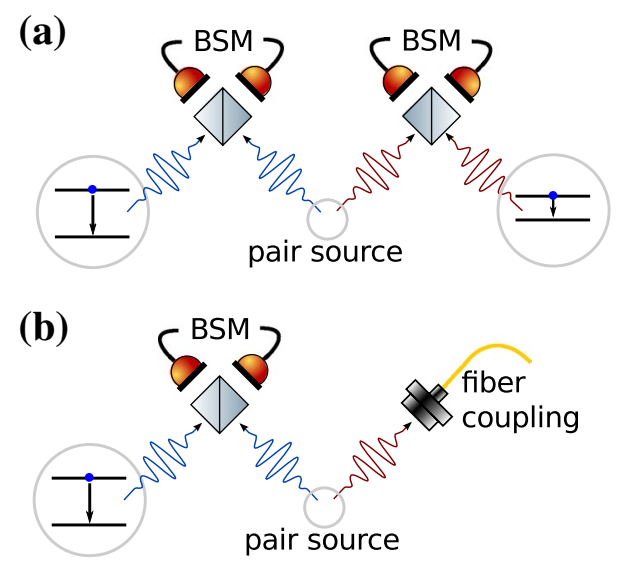

Fig. 1 Scheme of entanglement distribution via a two-color entangled photon source. a Two Bell-state measurements (BSM) on photons from two stationary qubit/flying qubit entangled systems and two photons from a two-color entangled photon source establish entanglement between two stationary qubits. b Similarly, a single Bellstate measurement on a photon from a stationary qubit/flying qubit entangled system and one photon from a two-color entangled photon source establish entanglement between the stationary qubit and a telecom photon

There are different possible collinear arrangements. Figure 2 shows the two-crystal Sagnac [9], the crossed-crystal [13] and the folded-sandwich configuration [14].

The rotation of the second crystal in the crossed-crystal configuration (Fig. 2b) can be replaced by a half-wave plate. Replacing the half-wave plate and the second crystal by a quarter-wave plate and a mirror yields the foldedsandwich scheme (Fig. 2c). Using only a single nonlinear crystal makes it considerably easier to avoid the leakage of which-crystal information. The folded-sandwich and the crossed-crystal configuration include a compensation crystal, which compensates the additional dispersion, whereas this is not needed in a two-crystal Sagnac configuration.

Together with the pump, any two-color entangled photon source involves three fields of widely different wavelengths. This imposes strong constraints on dispersion compensation. In particular, a source for generating entanglement in quantum hybrid systems should be easy to align, intrinsically stable and tunable in order to account for various transition frequencies. The Sagnac configuration requires a special three-color beam splitter and is very difficult to align [14]. The crossed-crystal configuration lacks the phase stability of the Sagnac configuration. Therefore, it is desirable to use the simpler folded-sandwich configuration. In this paper we show for the first time how the folded-sandwich can be used to generate highly non-degenerate photons. To this end, we slightly modify the foldedsandwich configuration. In contrast to previous work, we employ geometrical, i.e., wavelength-independent, polarization manipulation. (a) Sagnac two-crystal scheme
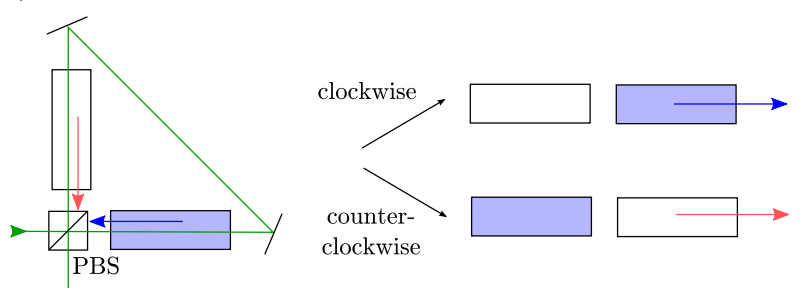

(b) Crossed-crystal scheme

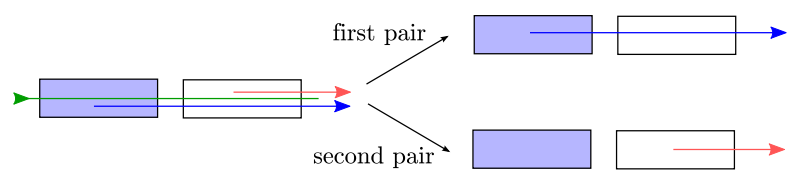

(c) (Geometrical) folded-sandwich scheme

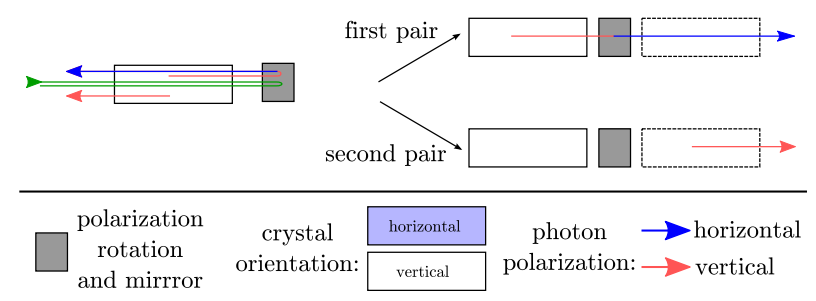

Fig. 2 Different schemes of two-color collinear entangled photon pair sources using type-0 down-conversion. A horizontally (vertically) polarized pump photon in an appropriate, i.e., horizontal (vertical), crystal creates horizontally (vertically) polarized signal and idler photons. No photons are created when pump polarization and crystal orientation are orthogonal. a The two possible paths in the two-crystal Sagnac configuration. In the clockwise and counterclockwise path, the photon pair is generated in the second crystal. In the (counter) clockwise path, the photon pair is generated vertically (horizontally) because the second crystal has horizontal (vertical) orientation. b The two paths in the crossed-crystal configuration. The first photon pair (vertically polarized) is created in the first crystal and accumulates an extra phase while passing through the second crystal. The second photon pair is created in the last crystal, just as the counterclockwise photon in the Sagnac configuration. $\mathbf{c}$ Folded-sandwich configuration. The polarization rotation element and a mirror (both depicted as one gray box) rotate and reflect the first pair. This is equivalent to the first pair in the crossed-crystal scheme, only that instead of the crystal the light polarization is rotated. In the folded-sandwich configuration, the second crystal is the mirrored first crystal. The (diagonal or unpolarized) pump beam is depicted in green

Here, we target for a two-color entangled photon source at $\lambda_{\mathrm{s}}=894.3 \mathrm{~nm}$ and $\lambda_{i}=1313.1 \mathrm{~nm}$. These two wavelengths correspond to the Cs D1 line and the telecom O-band, respectively. The former has been chosen on the one hand as a convenient standard atomic transition. On the other hand, it is also accessible with excitonic transitions in InGaAs quantum dots [15]. The source is thus applicable for quantum hybrid systems $[16,17]$ involving atomic species, semiconductor quantum dots or molecules as well as long-distance transfer via optical fibers. Here we aim on long-distance transfer of qubits from quantum dots. 


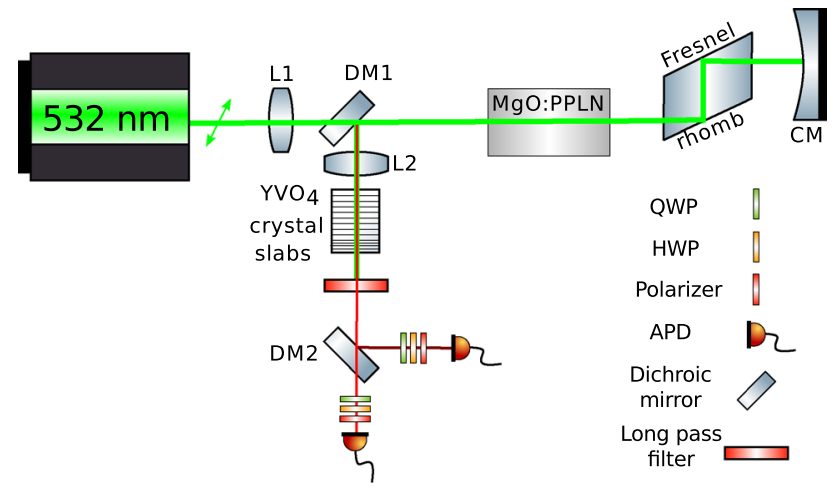

Fig. 3 Setup based on one periodically poled lithium niobate crystal (ppLN) doped with approximately $5 \%$ magnesium oxide $(\mathrm{MgO})$, of length $L=40 \mathrm{~mm}$. The crystal temperature is controlled via a crystal oven (not shown). The Fresnel rhomb acts as a geometrical quarter-wave plate. The concave mirror $(\mathrm{CM})$ reflects the light back into the crystal. The focusing (L1) and collimation (L2) lenses adjust the beam width. The first dichroic mirror (DM1) separates pump and down-converted photons. The second dichroic mirror (DM2) separates signal and idler photons. The phase compensation crystal consists of several $\mathrm{YVO}_{4}$ slabs to allow for dispersion control for a broad range of wavelengths. Both crystal ovens are omitted in this figure [18]

\section{Setup}

The setup is shown in Fig. 3. It resembles a folded-sandwich, but the specifically tailored achromatic quarter-wave plate which is used in [14] is replaced by a wavelengthindependent Fresnel rhomb. The Fresnel rhomb yields a $\lambda / 4$ phase shift after two internal reflections. A diagonal polarized pump laser $(532 \mathrm{~nm} \mathrm{cw}$ ) is focused into the nonlinear crystal (length $40 \mathrm{~mm}$, facet $4 \mathrm{~mm} \times 1 \mathrm{~mm}$, type-0 phase matching, grating period of $7 \mu \mathrm{m}$, multiple gratings, HC Photonics Corp.). In this first pass, the vertical component of the diagonal pump beam may create a vertically polarized pair. This pair and the pump beam propagate through the Fresnel rhomb. The Fresnel rhomb is oriented at $45^{\circ}$, such that it acts on the vertically polarized photons as a quarter-wave plate. The vertical pair leaves the rhomb circularly polarized. The diagonal pump beam passes the Fresnel rhomb without modification. The concave mirror (CM) reflects the light back onto the Fresnel rhomb and into the crystal. The mirror is adjusted to reflect the light into the same spatial mode. On the way back, the Fresnel rhomb rotates the circularly polarized pair into a horizontally polarized pair.

The diagonal pump beam and the horizontally polarized pair pass the nonlinear crystal a second time. Again, the vertical part of the diagonal pump beam may create a vertically polarized pair. The pump power is chosen such that the probability for creating more than one pair per double-pass is low. The pump polarization is chosen such that the count rate is the same for detection in the horizontal and vertical basis. Slight differences in the creation probability in the first and the second pass can be compensated by adjusting the pump polarization. The pump beam and the created photons are separated at the dichroic mirror (DM1). The photon pair is then collimated (L2) and directed onto a compensation crystal. Finally, signal and idler photons are separated at a dichroic mirror (DM2). In each of the separate arms, wave plates and polarizers allow for measurement of different polarization states. Each arm is coupled to a single-mode fiber. The fibers can either be connected to a spectrograph or to avalanche photodiodes (APDs) for spectral or coincidence measurements.

In order to generate an entangled state of the form

$|\psi\rangle=\frac{1}{\sqrt{2}}\left(|V V\rangle+e^{i \phi}|H H\rangle\right)$.

it is required to compensate for the different dispersive characteristics accumulated by the first pair due to the extra pass through the rhomb and the nonlinear crystal. Otherwise, this accumulated which-crystal information would diminish the entanglement.

The extra phase $\phi$ of the first pair $|H H\rangle$ is given by

$\phi\left(\lambda_{\mathrm{s}}, \lambda_{i}, T\right)=\left(\frac{n_{\mathrm{o}}\left(\lambda_{\mathrm{s}}, T\right)}{\lambda_{\mathrm{s}}}+\frac{n_{\mathrm{o}}\left(\lambda_{\mathrm{i}}, T\right)}{\lambda_{i}}\right) L_{\text {crystal }}$

$+\left(\frac{n_{\mathrm{r}}\left(\lambda_{\mathrm{s}}\right)}{\lambda_{\mathrm{s}}}+\frac{n_{\mathrm{r}}\left(\lambda_{i}\right)}{\lambda_{i}}\right) 2 L_{\mathrm{rhomb}}$

where $n_{\mathrm{r}}$ is the refractive index of the Fresnel rhomb (BK7 glass of length $L_{\mathrm{rhomb}}$ ) and $n_{\mathrm{o}}$ is the refractive index of the ordinary (horizontal) nonlinear crystal axis of length $L_{\text {crystal. }}$ The refractive index of the nonlinear crystal depends on the crystal temperature $T$ and the wavelengths of the down-converted signal and idler photons $\lambda_{\mathrm{s} / i}$.

In our experiment, we chose undoped $\mathrm{YVO}_{4}$ as a birefringent compensation crystal. A crystal of length $\tilde{L}$ adds a phase of

$$
\begin{aligned}
\tilde{\phi}\left(\lambda_{\mathrm{s}}, \lambda_{i}, T\right)= & \left(\frac{\tilde{n}_{\mathrm{o}}\left(\lambda_{\mathrm{s}}, T\right)}{\lambda_{\mathrm{s}}}+\frac{\tilde{n}_{\mathrm{o}}\left(\lambda_{i}, T\right)}{\lambda_{i}}\right. \\
& \left.-\frac{\tilde{n}_{\mathrm{e}}\left(\lambda_{\mathrm{s}}, T\right)}{\lambda_{\mathrm{s}}}-\frac{\tilde{n}_{\mathrm{e}}\left(\lambda_{i}, T\right)}{\lambda_{i}}\right) \tilde{L},
\end{aligned}
$$

where $\tilde{n}_{\mathrm{o} / \mathrm{e}}$ is the refractive index of the ordinary and extraordinary axis of $\mathrm{YVO}_{4}$.

The flat phase condition for optimum compensation with the two different photon wavelengths $\lambda_{\mathrm{s}}$ and $\lambda_{i}$ reads:

$\left.\frac{\mathrm{d}}{\mathrm{d} \lambda}(\phi+\tilde{\phi})\right|_{\lambda_{\mathrm{s}, i}}=0$. 
Table 1 Reported refractive index difference $\Delta n=n_{\mathrm{e}}-n_{\mathrm{o}}$ of the ordinary and extraordinary axis of $\mathrm{YVO}_{4}$ from different sources and the calculated corresponding length of compensation crystal for $\lambda_{\mathrm{s}}=894.3 \mathrm{~nm}$ and $\lambda_{i}=1313.1 \mathrm{~nm}$

\begin{tabular}{llll}
\hline Source & $\Delta n_{\mathrm{s}}$ & $\Delta n_{i}$ & $\mathrm{~L}(\mathrm{~mm})$ \\
\hline Manufacturer (Foctek) [19] & 0.211014 & 0.205271 & 138.7 \\
Zelmon et al. [20] & 0.211408 & 0.205448 & 154.0 \\
Sato et al. [21] & 0.211750 & 0.205685 & 172.0 \\
Handbook of optics [22] & 0.209959 & 0.204678 & 178.8 \\
\hline
\end{tabular}

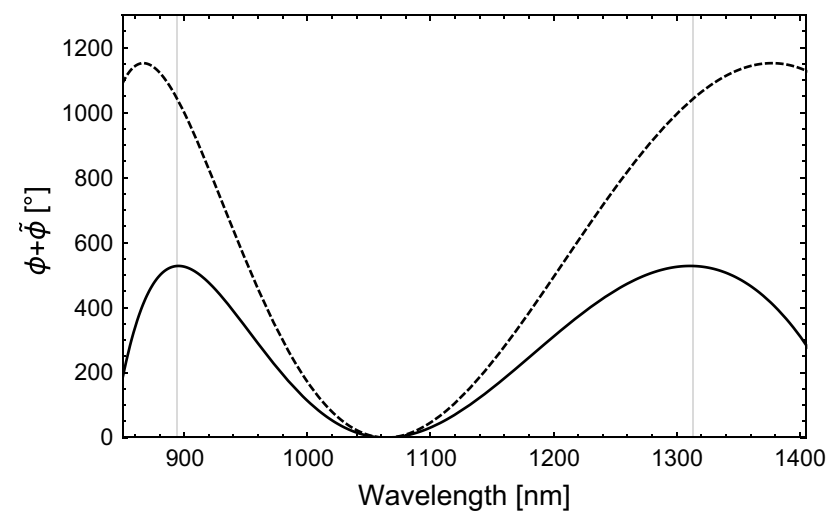

Fig. 4 Calculated phase $\phi+\tilde{\phi}$ as a function of the wavelength for two different lengths of the compensation crystal (constant offset subtracted). A flat phase is obtained for $\tilde{L}=154 \mathrm{~mm}$ (solid line) for the two target wavelengths $\lambda_{\mathrm{s}}=894.3 \mathrm{~nm}$ and $\lambda_{i}=1313.1 \mathrm{~nm}$ (vertical lines). The plateaus of the flat phases are shifted considerably for $\tilde{L}=153 \mathrm{~mm}$ (dashed line). Based on refractive index data reported by Zelmon et al. [20]

There are different values for the birefringent properties of $\mathrm{YVO}_{4}$ in the literature. Table 1 lists the refractive index difference of the ordinary and extraordinary axis of $\mathrm{YVO}_{4}$ and the calculated length of a compensation crystal for the wavelengths $\lambda_{\mathrm{s}}=894.3 \mathrm{~nm}$ and $\lambda_{i}=1313.1 \mathrm{~nm}$ for different sources. For the values that Zelmon et al. provide [20], Fig. 4 shows the calculated phase $\phi+\tilde{\phi}$ as a function of the wavelength for two different lengths of the compensation crystal. The two target wavelengths $\lambda_{\mathrm{s}}=894.3 \mathrm{~nm}$ and $\lambda_{i}=1313.1 \mathrm{~nm}$ are plotted as vertical lines.

For $\pm 1 \mathrm{~mm}$ crystal length, the two flat phase positions vary by $\pm 50 \mathrm{~nm}$. Thus with adding or removing additional few mm slabs of $\mathrm{YVO}_{4}$, the total compensation can be tuned by several hundred nanometers. Each slab introduces losses. Therefore, it is convenient to use AR-coated and as few slabs as possible. In this experiment, the refractive indices had to be examined first; therefore, a flexible set of seven slabs was chosen, which reduced the transmission to $82.64 \%$.

Finally, Fig. 5 demonstrates the tuning capability of our source. The crystal oven of the nonlinear crystal can be heated up to $160^{\circ} \mathrm{C}$. The measured tuning range of

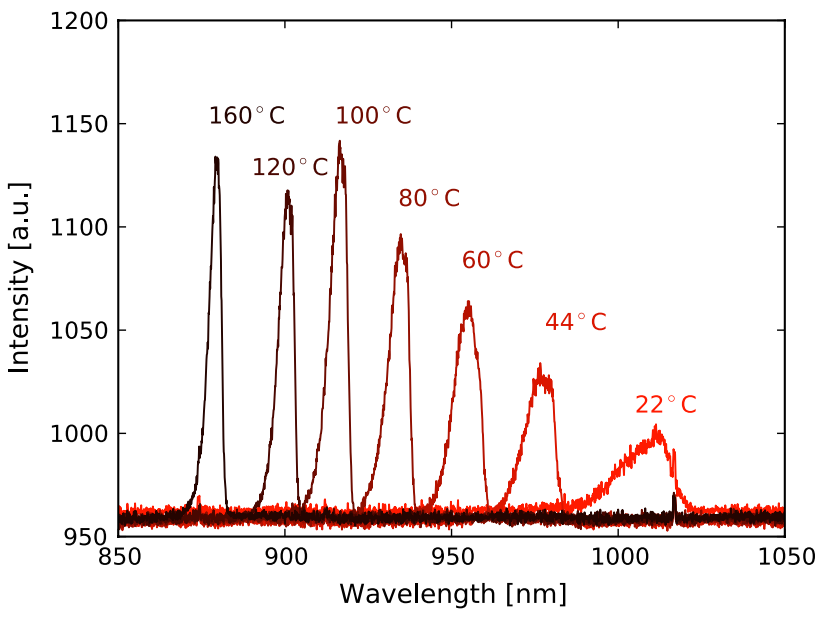

Fig. 5 Spectra of signal photons for different temperatures of the nonlinear crystal. The decreasing intensities with higher temperatures are due to decreasing sensitivity of the spectrometer CCD camera. At $1064 \mathrm{~nm}$, the signal and idler photons are degenerate

the signal photon wavelength for the chosen $7 \mu \mathrm{m}$ grating extends from 870 to $1100 \mathrm{~nm}$; this is roughly $230 \mathrm{~nm}$. The corresponding idler wavelength spans from 1124 to $1345 \mathrm{~nm}$ (not shown). The measurement was performed without compensation crystal.

\section{Determining the optimal crystal length}

The wave plates in each arm (after DM2 in Fig. 3) can be rotated such that photon coincidences in different bases can be measured. Both polarizers are fixed to horizontal polarization, such that in each arm the basis $|H\rangle$ is measured for a half-wave plate position of $\Theta_{\mathrm{HWP}}=0$. Rotating the half-wave plate to $\Theta_{\mathrm{HWP}}= \pm 22.5^{\circ}$, the diagonal $|D\rangle$ and anti-diagonal $|A\rangle$ basis is measured, respectively. Adding a quarter-wave plate, the left $|L\rangle$ and right $|R\rangle$ basis can be measured. Each arm can be set to an individual polarization. For example, setting both arms to left-circularly polarization is denoted $|L\rangle_{\text {signal }}|L\rangle_{\text {idler }}=|L L\rangle$ in the following.

The procedure to find the right compensation crystal relies on measuring polarization-sensitive coincidence counts as a function of the orientation of the half-wave plate $\theta_{\text {HWP }}$ in one arm. Fig. 6 shows such measurements in different bases combinations. The measured curve can be fitted to a sine function, and the visibility can be derived (see next Section for details). Then, thin slabs of $\mathrm{YVO}_{4}$ are added, until the visibility cannot be enhanced further. In this way, an optimum total length of the compensation crystal can be found.

Unfortunately, tuning by adding thin slabs of additional compensation crystals does not provide sufficient accuracy. 

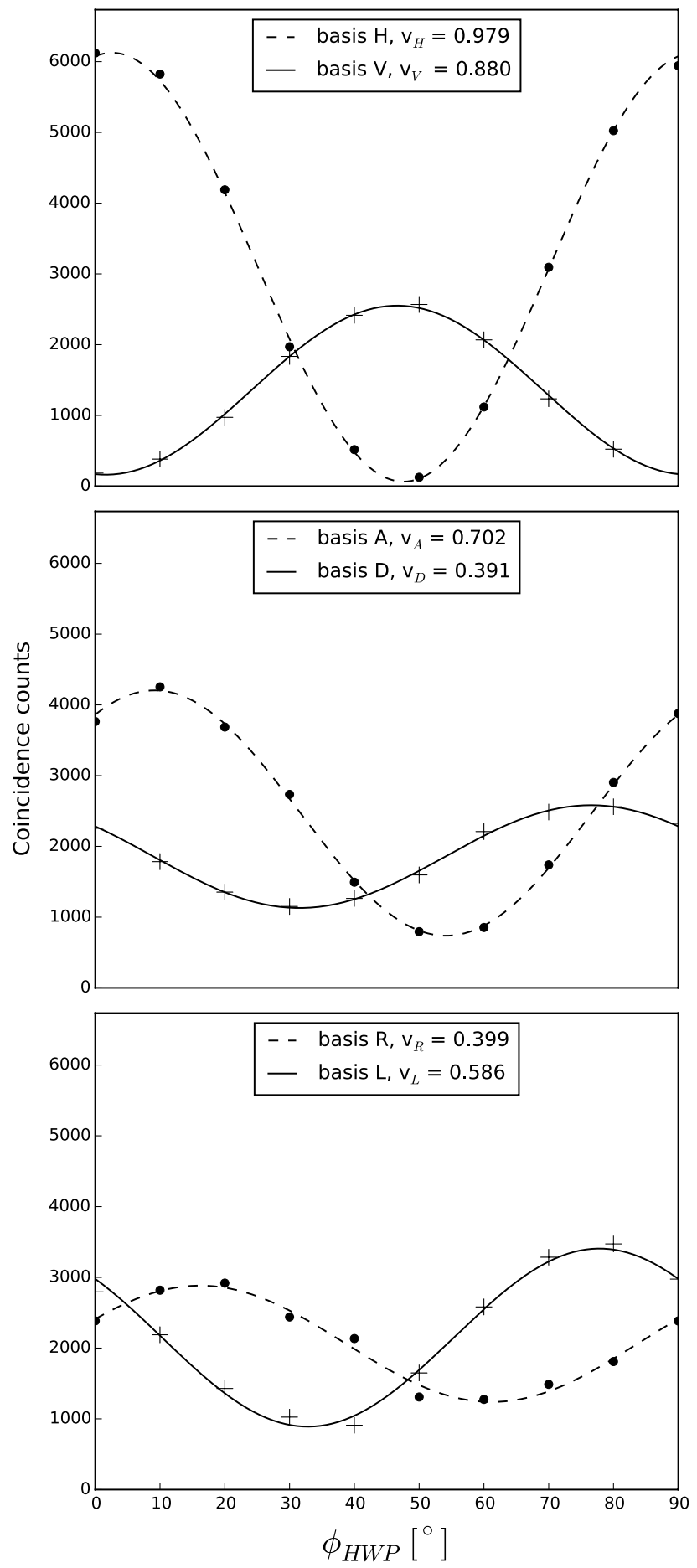

Fig. 6 Measured coincidence counts as a function of the orientation of the half-wave plate in different bases combinations. The dashed and solid lines are fits to sine functions. The resulting visibilities $v_{i}$ from Eq. (15) are indicated

Therefore, a fine tuning of the phase between 0 and $\pi$ to generate a specific Bell state is necessary.

In order to do this, we changed the temperature of a $30 \mathrm{~mm}$ compensation crystal slab. Figure 7 shows the measured

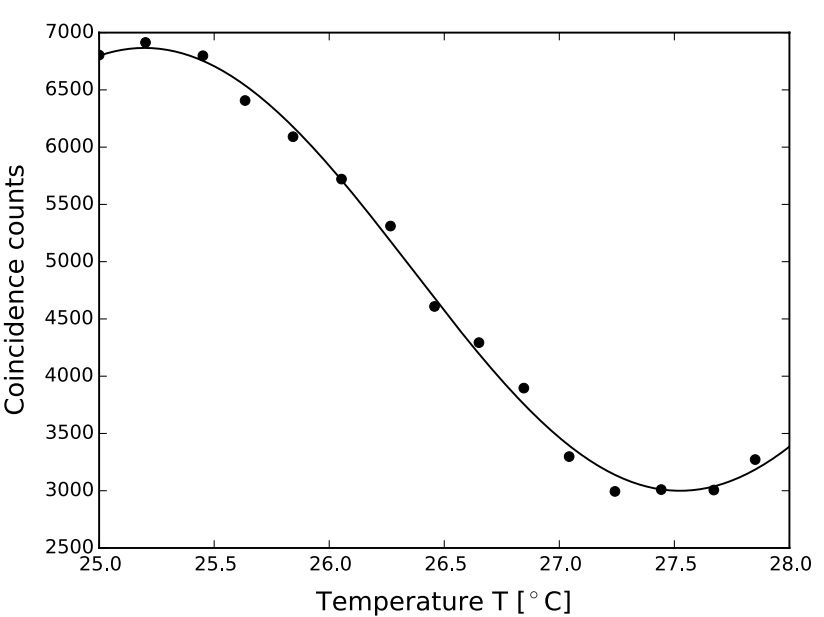

Fig. 7 Measured coincidence counts in the $|A A\rangle$ basis (both polarizers anti-diagonal) as a function of the temperature of a $30 \mathrm{~mm}$ compensation crystal slab. The total crystal length is $153 \mathrm{~mm}$

coincidence counts between signal and idler photons in the $|A A\rangle$ basis as a function of the temperature of the compensation crystal. We find a phase shift of $\pi$, i.e., between two different Bell states, for $\sim 2.4^{\circ} \mathrm{C}$ temperature difference.

For the wavelengths $\lambda_{\mathrm{s}}=894.3 \mathrm{~nm}$ and $\lambda_{i}=1313.1 \mathrm{~nm}$ we find a optimal total crystal length of $L=153 \mathrm{~mm}$. The total length was composed of 7 slabs $\mathrm{YVO}_{4}$ of $2 \mathrm{~cm}$ length and one slab of $1 \mathrm{~cm}, 2 \mathrm{~mm}$ and $1 \mathrm{~mm}$ length. The total length is close to some of the reported data on the refractive index of $\mathrm{YVO}_{4}$ in the literature [20], even though they investigated $0.5 \%$ Nd-doped $\mathrm{YVO}_{4}$. However, it deviates from the value reported by other studies [21], as well as from the manufacturer specification (Foctek Inc.) [19].

With the experimentally determined crystal parameters, it is in principle straightforward to estimate the compensation crystal length also for other pairs of wavelengths. Additional crystal slabs can be added or removed allowing for a wide tuning range.

\section{Verifying entanglement}

To quantify the degree of entanglement for optimized conditions, we measured the $\left|\phi^{+}\right\rangle$Bell-state fidelity $F_{\phi^{+}}$of the created state $\rho$,

$F_{\phi^{+}}=\left\langle\phi^{+}|\rho| \phi^{+}\right\rangle$.

A fidelity $F_{\phi^{+}}>\frac{1}{2}$ indicates entanglement [23]. In order to relate the fidelity to the coincidence probabilities, we use the two-photon polarization basis states $\left|u_{1}\right\rangle=|H H\rangle$, $\left|u_{2}\right\rangle=|H V\rangle,\left|u_{3}\right\rangle=|V H\rangle,\left|u_{4}\right\rangle=|V V\rangle$. After expanding $\rho$ in this basis and using

$\left|\phi^{+}\right\rangle=\frac{1}{\sqrt{2}}\left(\left|u_{1}\right\rangle+\left|u_{4}\right\rangle\right)$, 
we get

$$
\begin{aligned}
F_{\phi^{+}} & =\sum_{k, l=1}^{4} \rho_{k l}\left\langle\phi^{+} \mid u_{k}\right\rangle\left\langle u_{l} \mid \phi^{+}\right\rangle \\
& =\frac{1}{2}\left(\rho_{11}+\rho_{14}+\rho_{41}+\rho_{44}\right),
\end{aligned}
$$

where $\rho_{k l}=\left\langle u_{k}|\rho| u_{l}\right\rangle$. The diagonal elements $\rho_{11}$ and $\rho_{44}$ are the probabilities $P_{H}$ and $P_{V}$ of finding a photon pair in the states $|H H\rangle$ and $|V V\rangle$, respectively. The off-diagonal elements can be expressed with the help of the diagonal and circular basis,

$$
\begin{aligned}
P_{D}+P_{A} & =\langle D D|\rho| D D\rangle+\langle A A|\rho| A A\rangle \\
& =\frac{1}{2}\left(1+\rho_{14}+\rho_{41}+\rho_{23}+\rho_{32}\right),
\end{aligned}
$$

$$
P_{R}+P_{L}=\frac{1}{2}\left(1-\rho_{14}-\rho_{41}+\rho_{23}+\rho_{32}\right)
$$

and thus

$$
P_{D}+P_{A}-\left(P_{R}+P_{L}\right)=\rho_{14}+\rho_{41} \text {. }
$$

We therefore obtain the fidelity as a function of the probabilities in the three different bases,

$F_{\phi^{+}}=\frac{1}{2}\left[P_{D}+P_{A}-\left(P_{R}+P_{L}\right)+P_{H}+P_{V}\right]$.

These probabilities can be measured as they are connected to the coincidence count rates $N$,

$P_{i}=\frac{N_{i i}}{N_{i i}+N_{i j}+N_{j j}+N_{j i}}=\frac{N_{i i}}{N_{\mathrm{tot}}}$,

where $i$ refers to the one-photon polarization state orthogonal to $j$, that is $i / j=H / V, D / A$, or $L / R$. After introducing

$V_{i j}=\frac{N_{i i}+N_{j j}-\left(N_{i j}+N_{j i}\right)}{N_{i i}+N_{j j}+N_{i j}+N_{j i}}=\frac{2\left(N_{i i}+N_{j j}\right)}{N_{\text {tot }}}-1$

Equation (11) yields the alternative representation [14]

$F_{\phi^{+}}=\frac{1}{4}\left(1+V_{H V}+V_{D A}-V_{L R}\right)$.

To determine the fidelity, we measured the visibilities

$v_{i}=\frac{N_{i i}-N_{i j}}{N_{i i}+N_{i j}}=\frac{A_{i}}{C_{i}}$

in the three different bases, as shown in Fig. 6. In the measurement, the basis in one arm is fixed, while the basis in the other arm is rotated between $i$ and its orthogonal counter part $j$. The visibilities are then fitted with a sine function $A_{i} \sin \left(4 \phi_{\mathrm{HWP}}+\right.$ const. $)+C_{i}$. Since $C_{i}=\left(N_{i i}+N_{i j}\right) / 2$ and $A_{i}=\left(N_{i i}-N_{i j}\right) / 2$, we have
$V_{i j}=\frac{A_{i}+A_{j}}{C_{i}+C_{j}}$.

With this, we find a fidelity of $F_{\phi^{+}}=0.753 \pm 0.021$ for our source at a photon pair generation rate of $5.8 \mathrm{Mcps} / \mathrm{mW}$ and spectral linewidths of $560 \mathrm{GHz}$, which corresponds to 1.5 and $3.3 \mathrm{~nm}$ at $\lambda_{\mathrm{s}}=894.3 \mathrm{~nm}$ and $\lambda_{i}=1313.1 \mathrm{~nm}$, respectively. In order to match the bandwidth of the photons to stationary quantum systems, additional spectral filtering is required. External volume Bragg gratings and etalons have been used for this purpose [24, 25] starting with very similar bandwidths [25] as in our source. It is apparent that the initial spectral brightness has to be as large as possible in order to provide a reasonable photon pair rate after the filters. These values were calculated from a count rate of $215 \mathrm{kcts} / \mathrm{s}$ at $370 \mu \mathrm{W}$ and a detection efficiency of 0.1 . The error for the fidelity was calculated from the correlated errors of the visibilities (shown in Fig. 6). The uncertainties of the individual data points that stem from accidental coincident are negligibly small. Therefore, the error was estimated from the fit itself. The differences in the visibilities of orthogonal bases are due to a variety of reasons, such as the displacement differences in beam waists of $\mathrm{H}$ and $\mathrm{V}$ beam and a non-perpendicular incident on one of the compensation crystals causing wavelength-dependent diffraction. The limited visibility can be explained by temperature fluctuations of the compensation crystals. The total length of the compensation crystal is $L=153 \mathrm{~mm}$, but only a small fraction $(3 \mathrm{~cm})$ of the crystal is temperaturestabilized inside the crystal oven. Small changes in temperature modify the optical path lengths of extraordinarily and ordinarily polarized photons and lead to a fluctuating phase. For our configuration, the temperature fluctuations at the compensation crystals of $\pm 1 \mathrm{~K}$ are the strongest factor that reduces the fidelity (see also [14]). Temperature control and stabilization will also allow for better adjustment of the refractive indices, which in turn will enhance the visibility. With an improved temperature, stabilization of $0.1 \mathrm{~K}$ and a thermally isolated housing of the compensation crystals fidelities above $95 \%$ should be possible (as estimated in [14]).

\section{Conclusion}

We demonstrated a novel folded-sandwich scheme for the generation of two-color entangled photons which uses a Fresnel rhomb as a geometrical quarter-wave plate. By this, all optical components are more easily adapted to wide combinations of wavelengths. For example, no three-color beam splitters as in a Sagnac configuration are required. Adjusting the compensation crystal length offers a tuning 
capability over more than $100 \mathrm{~nm}$. Our source is a viable tool to provide highly non-degenerate entangled photons for quantum hybrid systems, in particular when solid-state emitters with an a priori unpredictable transition frequency are involved.

Acknowledgments This work was funded by BMBF (Q.comH). Funding by DFG through SFB 787 is acknowledged by O.D. O.D. likes to thank Sven Ramelow, Fabian Steinlechner and Amir Moqanaki for their hospitality during his stay in Vienna and their continuous input and support on the experimental design. This work was also supported by project EMPIR 14IND05 MIQC2 (the EMPIR initiative is co-funded by the European Union's Horizon 2020 research and innovation programme and the EMPIR Participating States).

\section{References}

1. H.J. Briegel, W. Dür, J.I. Cirac, P. Zoller, Phys. Rev. Lett. 81(26), 5932 (1998). doi:10.1103/PhysRevLett.81.5932

2. J. Volz, M. Weber, D. Schlenk, W. Rosenfeld, J. Vrana, K. Saucke, C. Kurtsiefer, H. Weinfurter, Phys. Rev. Lett. 96(3), 030404 (2006). doi:10.1103/PhysRevLett.96.030404

3. B.B. Blinov, D.L. Moehring, L.M. Duan, C. Monroe, Nature 428(6979), 153 (2004). doi:10.1038/nature02377. http://www. nature.com/nature/journal/v428/n6979/full/nature02377.html

4. W.B. Gao, P. Fallahi, E. Togan, J. Miguel-Sanchez, a Imamoglu, Nature 491(7424), 426 (2012). doi:10.1038/nature11573

5. E. Togan, Y. Chu, A.S. Trifonov, L. Jiang, J. Maze, L. Childress, M.V.G. Dutt, A.S. Srensen, P.R. Hemmer, A.S. Zibrov, M.D. Lukin, Nature 466(7307), 730 (2010). doi:10.1038/nature09256. http://www.nature.com/nature/journal/v466/n7307/full/ nature09256.html

6. A. Wallraff, D.I. Schuster, A. Blais, L. Frunzio, R.S. Huang, J. Majer, S. Kumar, S.M. Girvin, R.J. Schoelkopf, Nature 431(7005), 162 (2004). doi:10.1038/nature02851. http://www. nature.com/nature/journal/v431/n7005/full/nature02851.html

7. J. Hofmann, M. Krug, N. Ortegel, L. Gérard, M. Weber, W. Rosenfeld, H. Weinfurter, Science 337(6090), 72 (2012). doi:10.1126/science.1221856. http://www.sciencemag.org/ content $/ 337 / 6090 / 72$

8. H. Bernien, B. Hensen, W. Pfaff, G. Koolstra, M.S. Blok, L. Robledo, T.H. Taminiau, M. Markham, D.J. Twitchen, L. Childress, R. Hanson, Nature 497(7447), 86 (2013). doi:10.1038/ nature12016. http://www.nature.com/nature/journal/v497/n7447/ abs/nature12016.html

9. T.E. Stuart, J.A. Slater, F. Bussières, W. Tittel, Phys. Rev. A 88(1), (2013). doi:10.1103/PhysRevA.88.012301

10. P.G. Kwiat, K. Mattle, H. Weinfurter, A. Zeilinger, A.V. Sergienko, Y. Shih, Phys. Rev. Lett. 75(24), 4337 (1995). doi:10.1103/PhysRevLett.75.4337
11. M. Pelton, P. Marsden, D. Ljunggren, M. Tengner, A. Karlsson, A. Fragemann, C. Canalias, F. Laurell, Opt. Express 12(15), 3573 (2004). doi:10.1364/OPEX.12.003573. http://www.opticsexpress.org/abstract.cfm?URI=OPEX-12-15-3573

12. M. Hentschel, H. Hübel, A. Poppe, A. Zeilinger, Opt. Express 17(25), 23153 (2009). http://www.opticsinfobase.org/abstract. cfm?uri=oe-17-25-23153

13. P. Trojek, H. Weinfurter, Appl. Phys. Lett. 92(21), 211103 (2008). doi:10.1063/1.2924280

14. F. Steinlechner, S. Ramelow, M. Jofre, M. Gilaberte, T. Jennewein, M.W. Mitchell, V. Pruneri, J.P. Torres, Opt. Express 21(10), 11943 (2013). doi:10.1364/OE.21.011943. http://www. opticsinfobase.org/abstract.cfm?URI=oe-21-10-11943

15. F. Ding, R. Singh, J.D. Plumhof, T. Zander, V. Kápek, Y.H. Chen, M. Benyoucef, V. Zwiller, K. Dörr, G. Bester, A. Rastelli, O.G. Schmidt, Phys. Rev. Lett. 104(6), 067405 (2010). doi:10.1103/ PhysRevLett.104.067405

16. N. Akopian, L. Wang, A. Rastelli, O.G. Schmidt, V. Zwiller, Nat Photon 5(4), 230 (2011). doi:10.1038/nphoton.2011.16. http:// www.nature.com/nphoton/journal/v5/n4/full/nphoton.2011.16. html

17. P. Siyushev, G. Stein, J. Wrachtrup, I. Gerhardt, Nature 509(7498), 66 (2014). doi:10.1038/nature13191. http://www. nature.com/nature/journal/v509/n7498/full/nature13191.html

18. A. Franzen, ComponentLibrary: a free vector graphics library for optics. In: Licensed under a Creative Commons AttributionNonCommercial 3.0 Unported License. http://www.gwoptics. org/ComponentLibrary/

19. Foctek Photonics, Inc. YVO4. http://www.foctek.net/products/ YVO4.htm

20. D.E. Zelmon, J.M. Northridge, D. Perlov, Appl. Opt. 49(4), 644 (2010). doi:10.1364/AO.49.000644. http://ao.osa.org/abstract. cfm?URI=ao-49-4-644

21. Y. Sato, T. Taira, Opt. Mater. Express 4(5), 876 (2014). doi:10.1364/OME.4.000876. http://www.opticsinfobase.org/ ome/abstract.cfm?URI=ome-4-5-876

22. M. Bass, C. DeCusatis, J. Enoch, V. Lakshminarayanan, G. Li, C. MacDonald, V. Mahajan, E.V. Stryland, Handbook of Optics, Third Edition Volume IV: Optical Properties of Materials, Nonlinear Optics, Quantum Optics (McGraw Hill Professional, 2009)

23. A.G. White, A. Gilchrist, G.J. Pryde, J.L. O'Brien, M.J. Bremner, N.K. Langford, JOSA B 24(2), 172 (2007). http:// www.opticsinfobase.org/abstract.cfm?uri=josab-24-2-172

24. A. Ahlrichs, C. Berkemeier, B. Sprenger, O. Benson, Appl. Phys. Lett. 103(24), 241110 (2013). doi:10.1063/1.4846316

25. C. Clausen, F. Bussières, A. Tiranov, H. Herrmann, C. Silberhorn, W. Sohler, M. Afzelius, N. Gisin, N. J. Phys. 16(9), 093058 (2014). doi:10.1088/1367-2630/16/9/093058. http://stacks.iop. $\mathrm{org} / 1367-2630 / 16 / \mathrm{i}=9 / \mathrm{a}=093058 ? \mathrm{key}=$ crossref. $1 \mathrm{a} 036 \mathrm{eff} 85981$ 726152473f2abf2f1f5 